\title{
Prevalência de alterações metabólicas em crianças e adolescentes com sobrepeso e obesidade: uma revisão sistemática
}

\author{
Prevalence of metabolic changes in children and adolescents: a systematic review
}

Lúcia Gomes Rodrigues ${ }^{1}$, Nina Pombo², Sérgio Koifman ${ }^{3}$

\section{RESUMO}

Objetivo: Descrever a prevalência de síndrome metabólica em crianças e adolescentes com sobrepeso ou obesidade bem como os critérios utilizados em sua classificação.

Fontes dos dados: Revisão sistemática realizada por meio de busca eletrônica nas bases de dados Pubmed e na Biblioteca Virtual em Saúde. Os critérios de inclusão adotados foram apresentar dados de prevalência de síndrome metabólica em crianças e adolescentes com sobrepeso e obesidade, sendo publicados em português, inglês, espanhol e francês. Foram excluídos artigos de revisão, comunicação breve e estudos em populações com doenças de base (genética, endócrina, imunológica, hipertensão primária e presença de acantose).

Síntese dos dados: Foram levantados 1.226 resumos, sendo selecionados 65 artigos para análise na íntegra, dos quais 46 atendiam os critérios mencionados no período de 2003 a 2009, representando cinco regiões geográficas: América do Norte (33\%), América do Sul (20\%), América Central (4\%), Ásia (30\%) e Europa (13\%). As prevalências descritas variaram de 2,1 a $58,3 \%$, sendo $31,2 \%$ a prevalência mediana. Houve divergência nos critérios, com 26 estudos usando os mesmos componentes (triglicerídeos, HDL, glicose, circunferência de cintura e pressão arterial), sem consenso nos pontos de corte adotados. Nos demais estudos, houve inclusão de glicemia pós-prandial, índice de massa corporal, colesterol, e índice HOMA-IR.

Conclusões: A prevalência descrita de síndrome metabólica em crianças e adolescentes na literatura apresentou uma ampla variabilidade, ocorrendo heterogeneidade na escolha

Instituição: Escola Nacional de Saúde Pública Sergio Arouca da Fundação Oswaldo Cruz (Fiocruz) e Escola de Nutrição da Universidade Federal do Estado do Rio de Janeiro (Unirio)

'Doutoranda em Saúde Pública e Meio Ambiente pela Escola Nacional de Saúde Pública (ENSP) da Fiocruz; Professora Adjunta da Escola de Nutrição da Unirio, Rio de Janeiro, RJ, Brasil

${ }^{2}$ Nutricionista Graduada pela Unirio, Rio de Janeiro, RJ, Brasil

${ }^{3}$ Pós-Doutorado pela McGill University, Montreal, Canadá; Pesquisador e Coordenador do Programa de Pós-Graduação em Saúde Pública e Meio Ambiente da ENSP/Fiocruz, Rio de Janeiro, RJ, Brasil das variáveis empregadas na definição dos componentes da doença, bem como nos pontos de corte adotados.

Palavras-chave: síndrome X metabólica; criança; adolescente; obesidade; prevalência.

\section{ABSTRACT}

Objective: To describe the prevalence of the metabolic syndrome in children and adolescents, as well as the adopted classification criteria.

Data source: Systematic review performed by electronic search on PubMed and Virtual Library in Health database. The inclusion criteria were: metabolic syndrome prevalence data in children and adolescents with overweight and obesity, and publications in Portuguese, English, Spanish and French. The exclusion criteria were review articles and short communications, investigations enrolling participants with genetic, endocrine and immunologic diseases, primary hypertension, and acanthosis nigricans.

Data synthesis: The review afforded 1.226 abstracts, being 65 selected to be read, 46 of them matched the aforementioned selection criteria and could be retrieved. They were published between 2003 and 2009 and represented five geographic regions: North America (33\%), South America (20\%), Central America (4\%), Asia (30\%) and Europe $(13 \%)$. The metabolic syndrome reported prevalence ranged from 2.1 to $58.3 \%$. The adopted criteria diverged among the studies, 26 of them used the same components (neutral fat, HDL, glucose, waist circumference and blood pressure), with a median prevalence of $31.2 \%$, without agreement on the 
chosen cut-off points. In the remaining studies, metabolic syndrome definition included glucose oral tolerance, body mass index, serum cholesterol, and HOMA-IR index.

Conclusions: The metabolic syndrome prevalence among children and adolescents with obesity or overweight reported in the literature showed a wide variability. There was heterogeneity, regarding born the variables chosen to define the presence of metabolic syndrome and their respective cut-off points.

Key-words: metabolic syndrome X; child; adolescent; obesity; prevalence.

\section{Introdução}

A Organização Mundial da Saúde (OMS) informou, em 2006, que aproximadamente 1,6 bilhão de adultos apresentam excesso de peso e, entre as crianças maiores de cinco anos, o problema atingiria cerca de 20 milhões. Esse agravo nutricional não é privilégio dos países desenvolvidos, pois seu aumento tem se dado de forma dramática em países em desenvolvimento.

A prevalência do excesso de peso vem aumentando de forma alarmante no Brasil nos últimos 30 anos. Em 1975, segundo o Estudo Nacional de Despesa Familiar (ENDEF), os homens apresentavam $18,6 \%$ de excesso de peso e as mulheres, 28,6\%. Em 2003, a Pesquisa de Orçamentos Familiares (POF) indicou um aumento dessa prevalência, atingindo $41 \%$ dos homens e 39,8\% das mulheres. O estudo sobre Vigilância de Fatores de Risco e Proteção para Doenças Crônicas por Inquérito Telefônico (VIGITEL), realizado desde 2006 pelo Ministério da Saúde (MS), confirma a tendência de crescimento do excesso de peso no país. Em 2008, esse problema atingia $43,3 \%$ dos adultos, sendo da ordem de $47,3 \%$ nos homens e $39,5 \%$ nas mulheres.

Essa mesma tendência vem sendo observada na população infantil. De acordo com o ENDEF, a prevalência de excesso de peso na faixa etária de dez a 19 anos era de 7,5\% nas meninas e de 3,9\% nos meninos. Na Pesquisa Nacional sobre Saúde e Nutrição (PNSN) houve um aumento para 13,2 e $8,3 \%$, respectivamente. Após um intervalo de 14 anos, a POF apresentou um crescimento desses indicadores, atingindo $18 \%$ do sexo masculino e $15,4 \%$ do feminino.

$\mathrm{O}$ aumento da gordura corporal, principalmente a visceral, tem sido associado ao desenvolvimento de doenças crônicas, como diabetes melito, dislipidemias e hipertensão arterial. A gordura visceral tem maior atividade lipolítica quando comparada à gordura subcutânea e, consequentemente, libera maior quantidade de ácidos graxos livres e glicerol, transportados diretamente para o fígado, promovendo uma série de alterações no metabolismo lipídico. Além disso, o tecido adiposo tem papel de destaque na patogênese da resistência à insulina (RI), devido à liberação de metabólitos e hormônios que afetam diferentes etapas da ação da insulina ${ }^{(1)}$.

A resistência à insulina está associada ao desenvolvimento de uma variedade de fatores de risco para doenças cardiovasculares, entre as quais se incluem a intolerância à glicose, a hipertensão arterial, a diminuição do HDL colesterol e elevação dos triglicerídeos, a lesão vascular, as alterações de coagulação e a inflamação. Esses fatores são considerados os principais mecanismos que implicam no desenvolvimento da chamada síndrome metabólica (SM), descrita por Reaven como Síndrome $\mathrm{X}^{(1,2)}$.

Várias organizações internacionais, como a OMS, o National Cholesterol Education Program (NCEP), o International Diabetes Federation (IDF), o American College of Endocrinology (ACE) e o European Group for the Study of Insulin Resistance (EGIR), têm proposto definições da SM para adultos usando diferentes componentes e pontos de corte na distribuição das variáveis empregadas para defini-la. Apesar das tentativas recentes de se chegar a um consenso sobre a melhor definição dessa síndrome, ainda existem divergências entre os componentes e os pontos de corte, o que pode dificultar a comparação das prevalências relatadas nos diferentes estudos ${ }^{(3)}$.

Em função do crescimento da obesidade e dos fatores de risco para doenças cardiovasculares entre crianças e adolescentes obesos, tem surgido interesse em investigar a presença de alterações metabólicas similares àquelas observadas na SM do adulto. O Bogalusa Heart Study conta com estudos longitudinais, com crianças e adolescentes entre cinco e 17 anos, com o objetivo de avaliar fatores de risco para doenças cardiovasculares nas primeiras décadas de vida. Os resultados sugerem que a duração da obesidade encontra-se diretamente associada à morbimortalidade por aquelas patologias. As crianças com excesso de peso apresentaram uma razão de chances de 2,4 vezes para a elevação de colesterol e pressão arterial diastólica, 4,5 vezes para o aumento da pressão arterial sistólica, 7,1 vezes para o aumento de triglicerídeos e 12,6 para insulina de jejum, encontrando-se pelo menos um fator de risco (dislipidemia, hiperinsulinemia ou hipertensão arterial) em $58 \%$ da população com índice de massa cosporal

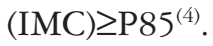

Sendo assim, vários estudos têm sido desenvolvidos com o intuito de diagnosticar a presença ou não da SM na 
população infantil ${ }^{(5,6)}$. Adicionalmente, ainda não existem critérios consistentes para diagnosticar a possível ocorrência dessa síndrome na população pediátrica, tanto nos componentes, como nos pontos de corte das variáveis utilizadas em sua definição ${ }^{(3)}$.

Devido ao aumento da obesidade nessa população e de suas consequências metabólicas, faz-se importante conhecer a dimensão desse problema, por meio de estudos que investiguem a prevalência de alterações metabólicas em crianças e adolescentes, bem como os critérios utilizados para sua classificação. Considerando-se a relevância do tema, o objetivo desta revisão sistemática foi descrever a prevalência de alterações metabólicas em crianças e adolescentes com sobrepeso ou obesidade relatada na literatura, bem como os critérios utilizados em sua classificação.

\section{Fonte dos dados}

Esse estudo consistiu em uma revisão sistemática realizada por meio de busca eletrônica nas bases de dados PubMed e Biblioteca Virtual em Saúde (BVS), que têm como fontes de informação as bases Lilacs, Medline, Biblioteca Cochrane e SciELO. Nas buscas, foi utilizado o seguinte unitermo: "prevalence metabolic syndrome children".

Foi desenvolvido um protocolo para seleção de resumos e posterior inclusão e exclusão dos artigos selecionados. Os estudos identificados foram selecionados a partir da leitura dos resumos, adotando-se como critério de inclusão aqueles que apresentassem informações relativas às estimativas de prevalência de SM em crianças e/ou adolescentes a partir de dados primários e publicação nos idiomas português, inglês, espanhol e francês.

Os seguintes critérios de exclusão foram adotados: artigos de revisão, comunicação breve, e aqueles realizados em população de estudo com doenças de base genética, endócrina, imunológica, hipertensão primária, ou presença de acantose. Não foi delimitado período das publicações, por se tratar de um tema recente no âmbito da faixa etária de interesse.

A busca dos artigos completos se deu por meio de revistas com acesso livre e gratuito online, pelo portal da Coordenação de Aperfeiçoamento de Pessoal de Nível Superior/Ministério da Educação (CAPES/MEC), por contato com os autores por correspondência eletrônica e busca no COMUT/SCAD.

Foi elaborada uma ficha para extração de informações dos artigos selecionados, na qual foram incluídos os seguintes dados: nomes dos autores, ano e local de publicação, população de estudo, tamanho e descrição da amostra, critérios de inclusão e exclusão, componentes da SM com pontos de corte adotados, prevalência das alterações metabólicas e da SM e análise estatística realizada.

\section{Síntese dos dados}

Inicialmente foram identificados os resumos de 1.226 estudos sobre SM infância e adolescência, sendo selecionados 65 artigos para leitura na íntegra. Após leitura dos artigos completos, 13 foram excluídos por não atenderem aos critérios de inclusão: ausência de estimativas de prevalência da SM na presença de sobrepeso ou obesidade (quatro artigos); publicações em outros idiomas, como japonês, romeno e polonês (três artigos); estudos realizados em crianças portadoras de diabetes melito, hipertensão arterial e acantose nigricans (três artigos); e publicações de comunicação breve e revisão (três artigos). Sendo assim, 46 artigos $^{(5,6,8-18,24-56)}$ fizeram parte dessa revisão, cujo fluxograma com as diferentes etapas relativas à estratégia adotada para identificação e inclusão dos estudos encontram-se retratadas na Figura 1.

Os artigos datavam do período de 2003 a 2009, sendo originários de cinco regiões geográficas: América do Norte $(33 \%)^{(5-7,14-16,24,29,35,38,40,42,49,51,56)}$, Ásia $(30 \%)^{(9-11,25,30,33,36,39,41,43,}$ 48,50,52,54) , América do Sul $(20 \%)^{(13,27,32,, 44-47,53,55)}$, Europa (13\%) $(12,18,26,28,31,34)$ e América Central $(4 \%)^{(8,37)}$. As prevalências relatadas apresentaram estimativas mais reduzidas na Europa (mediana de 22\%) $)^{(12,18,26,28,31,34)}$ e mais elevadas na América Central (mediana de $39 \%)^{(8,377)}$.

A magnitude de prevalências de SM, em crianças e adolescentes com sobrepeso e obesidade, foi divergente, variando de 2,1 a $58,3 \%{ }^{(30,44)}$ (Tabelas 1 e 2). A maior parte dos artigos selecionados (96\%) estabeleceu a presença de três ou mais componentes para o diagnóstico da SM. Quanto à população de estudo, 50\% (23 artigos) ${ }^{(7-10,13,15,16,18,26-36,44,44,53,56)}$ analisaram crianças e adolescentes conjuntamente, tendo $26 \%$ analisado somente criançass ${ }^{(11,12,14,24,38,40,41,43,45-47,55)}$ e $24 \%$ somente adolescentes ${ }^{(5,6,25,37,39,42,48,50,51,52,54)}$.

O critério diagnóstico da SM estabelecido pelo NCEP/ ATPIII para adultos, incluindo hipertrigliceridemia, redução do HDL-c, hiperglicemia de jejum, obesidade abdominal (circunferência de cintura - CC) e hipertensão arterial, foi utilizado em 28 artigos $(61 \%)^{(5-9,11-15,25,27,29,30,33,34,40-43,46-51,53,54)}$. Nesse grupo de artigos, a prevalência mediana encontrada foi de $31,2 \%$. Os outros estudos utilizaram diferentes componentes para caracterização da SM (Tabela 3).

Cerca de oito publicações basearam-se em dois ou mais conjuntos de variáveis para realizar o diagnóstico 


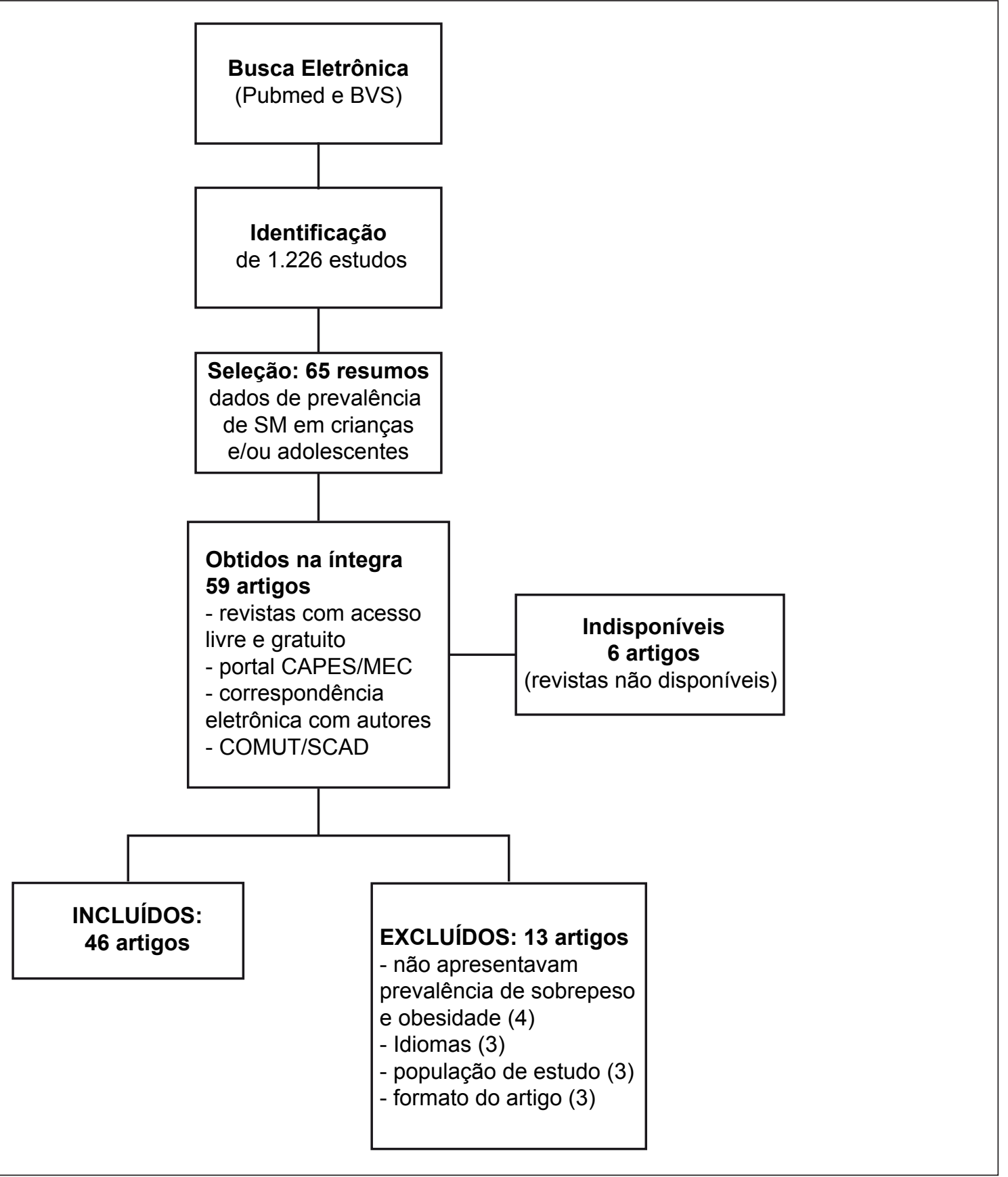

Figura 1 - Representação sistemática do método de busca e dos resultados obtidos.

da SM, tendo todas incluído alguma alteração no perfil lipídico (hipertrigliceridemia, hipercolesterolemia ou redução do HDL), homeostase anormal da glicose (hiperinsulinemia ou tolerância à glicose diminuída) e a presença de hipertensão arterial como componentes da $\mathrm{SM}^{(5,13,14,28,33,35,51,54,55)}$. Foram, ainda, citados outros componentes, como IMC $\operatorname{IM}^{(10,16,24,26,31,32,36,39,37,44,45,52,56)}$, colesterol $^{(10,33,36)}$ e índice Homeostatic Model Assessment for Insulin Resistance (HOMA-IR) ${ }^{(28,55)}$.
A divergência na escolha dos componentes para o diagnóstico da SM ocorreu também em relação à definição dos pontos de corte para cada uma dessas variáveis, com exceção do colesterol sérico, que apresentou o mesmo ponto de corte (percentil 95) nos estudos ${ }^{(10,33,36)}$ que utilizaram esse critério (Quadro 1).

Quanto ao HDL-c, não houve consenso tanto no ponto de corte, como na forma de apresentação. Alguns autores estabeleceram os limites em percentil de uma distribuição

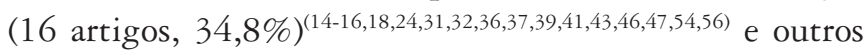


Tabela 1 - Prevalência de síndrome metabólica na infância e adolescência em populações referidas por unidades de saúde (ambulatórios e hospitais), revisão sistemática da literatura, 2003-2009

\begin{tabular}{|c|c|c|c|c|c|}
\hline Estudo & Ano & Local & População & $\mathbf{n}$ & Prevalência de SM (\%) \\
\hline \multirow[t]{4}{*}{ Boney et $a^{(24)}$} & \multirow[t]{4}{*}{2005} & \multirow{4}{*}{$\begin{array}{l}\text { Estados } \\
\text { Unidos }\end{array}$} & \multirow{4}{*}{$\begin{array}{l}\text { Crianças } 6 \text { anos, acompanhadas durante } \\
5 \text { anos, filhas de mães com e sem DG e } \\
\text { PN (GIG e AIG) }\end{array}$} & \multirow[t]{4}{*}{179} & GIG/DG - 50,0 \\
\hline & & & & & AIG/DG - 21,0 \\
\hline & & & & & GIG $-29,0$ \\
\hline & & & & & $\mathrm{AIG}-18,0$ \\
\hline Atabek et $a /^{(10)}$ & 2006 & Turquia & Crianças de 7 a 18 anos & 169 & 27,2 \\
\hline \multirow[t]{2}{*}{ Bueno et a/ ${ }^{(12)}$} & \multirow[t]{2}{*}{2006} & \multirow[t]{2}{*}{ Espanha } & Crianças obesas de $10,1 \pm 2,3$ anos. & \multirow[t]{2}{*}{103} & $29,9-$ Cook $^{(5)}$ \\
\hline & & & $\begin{array}{l}\text { Departamento de Pediatria Hospital de } \\
\text { Zaragoza }\end{array}$ & & $50,0-$ Ferranti $^{(6)}$ \\
\hline Esmaillzadeh et al(25) & 2006 & Irã & $\begin{array}{l}\text { Adolescentes de } 10 \text { a } 19 \text { anos que } \\
\text { participaram do estudo de dislipidemia }\end{array}$ & 3.036 & 56,6 \\
\hline López-Capapé et a/(26) & 2006 & Espanha & $\begin{array}{l}\text { Crianças e adolescentes de } 11,2 \pm 2,8 \\
\text { anos que se apresentaram para } \\
\text { avaliação da obesidade }\end{array}$ & 429 & 18,0 \\
\hline Quintos et al(7) & 2006 & $\begin{array}{l}\text { Estados } \\
\text { Unidos }\end{array}$ & $\begin{array}{l}\text { Crianças e adolescentes obesos de } 3 \text { a } \\
18 \text { anos, inscritos em programa de perda } \\
\text { de peso }\end{array}$ & 194 & 35,5 \\
\hline Buff et a/(27) & 2007 & Brasil & $\begin{array}{l}\text { Crianças e adolescentes, } 10,9 \pm 0,48 \\
\text { anos matriculados no Ambulatório de } \\
\text { Obesidade }\end{array}$ & 59 & 42,4 \\
\hline \multirow[t]{2}{*}{ Burrows et a/ ${ }^{(13)}$} & \multirow[t]{2}{*}{2007} & \multirow[t]{2}{*}{ Chile } & \multirow{2}{*}{$\begin{array}{l}\text { Crianças com sobrepeso ou obesidade, } \\
6 \text { e } 16 \text { anos, atendidos no Programa } \\
\text { Clínico de Obesidade }\end{array}$} & \multirow[t]{2}{*}{489} & $26,8-$ Cook $^{(5)}$ \\
\hline & & & & & $45,6-$ Ferranti $^{(6)}$ \\
\hline Ceballos et al(28) & 2007 & Espanha & $\begin{array}{l}\text { Crianças e adolescentes obesos, } 6 \text { e } 14 \\
\text { anos, da Endocrinologia Pediátrica }\end{array}$ & 97 & 18,6 a 34,0 \\
\hline \multirow[t]{2}{*}{ Dhuper et a/(29) } & \multirow[t]{2}{*}{2007} & \multirow{2}{*}{$\begin{array}{l}\text { Estados } \\
\text { Unidos }\end{array}$} & Crianças e adolescentes obesos & \multirow[t]{2}{*}{576} & \multirow[t]{2}{*}{44,6} \\
\hline & & & $\begin{array}{l}3 \text { e } 19 \text { anos, programa de obesidade de } \\
\text { um Hospital Universitário }\end{array}$ & & \\
\hline Serap et al(30) & 2007 & Turquia & $\begin{array}{l}\text { Crianças e adolescentes, } 6 \text { a } 16 \text { anos, } \\
\text { em Hospital Universitário }\end{array}$ & 284 & 2,1 \\
\hline Calcaterra et a/(31) & 2008 & Itália & $\begin{array}{l}\text { Crianças e adolescentes obesos } \\
\text { caucasianos, idade de } 11,2 \pm 3,4 \text { anos }\end{array}$ & 191 & 13,9 \\
\hline Carceres et al(32) & 2008 & Bolívia & $\begin{array}{l}\text { Crianças e adolescentes obesos, } \\
4 \text { a } 18 \text { anos }\end{array}$ & 61 & 36,1 \\
\hline \multirow[t]{2}{*}{ Çizmecioglu et a/(33) } & \multirow[t]{2}{*}{2008} & \multirow[t]{2}{*}{ Turquia } & \multirow{2}{*}{$\begin{array}{l}\text { Crianças e adolescentes obesos, } \\
2 \text { a } 18 \text { anos, buscando atenção de } \\
\text { Hospital Universitário }\end{array}$} & \multirow[t]{2}{*}{112} & $24,0-$ NCEP \\
\hline & & & & & $30,0-\mathrm{OMS}$ \\
\hline Kolsgaard et a ${ }^{(34)}$ & 2008 & Noruega & $\begin{array}{l}\text { Crianças e adolescentes obesos de Oslo, } \\
6 \text { a } 17 \text { anos, participantes de programa } \\
\text { de intervenção }\end{array}$ & 203 & 24,7 \\
\hline Lee et $a /^{(35)}$ & 2008 & $\begin{array}{l}\text { Estados } \\
\text { Unidos }\end{array}$ & $\begin{array}{l}\text { Crianças e adolescentes participantes de } \\
\text { estudos metabólicos }\end{array}$ & 251 & 24,0 a 51,0 \\
\hline Mimoun et a/(18) & 2008 & França & $\begin{array}{l}\text { Crianças e adolescentes obesos, } \\
2,5 \text { a } 18 \text { anos recrutados em três } \\
\text { hospitais-escola }\end{array}$ & 384 & 10,4 \\
\hline Sen et al(36) & 2008 & Turquia & $\begin{array}{l}\text { Crianças obesas, } 2 \text { a } 19 \text { anos, buscando } \\
\text { atenção de Hospital Universitário }\end{array}$ & 352 & 41,8 \\
\hline
\end{tabular}

SM: síndrome metabólica; DG: diabetes gestacional; PN: peso ao nascer; GIG/DG: grande para a idade gestacional e diabetes gestacional; AIG/ DG: adequado para a idade gestacional e diabetes gestacional; GIG: grande para a idade gestacional sem diabetes gestacional; AIG: adequado para a idade gestacional sem diabetes gestacional; NCEP: National Cholesterol Education Program; OMS: Organização Mundial da Saúde. 
Tabela 2 - Prevalência de síndrome metabólica na infância e adolescência em população geral (escolas, inquéritos de saúde), revisão sistemática da literatura, 2003-2009

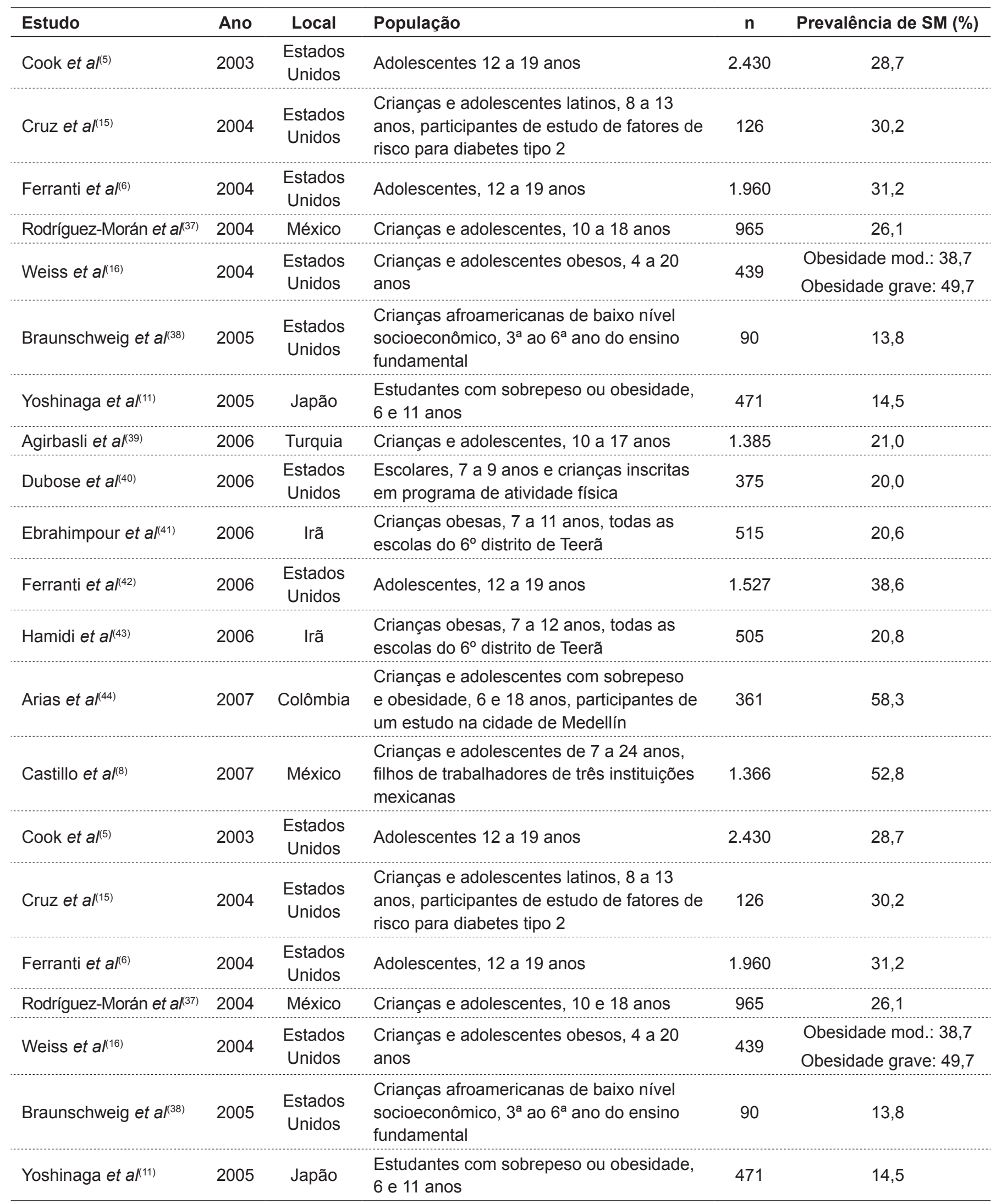


Continuação - Tabela 2

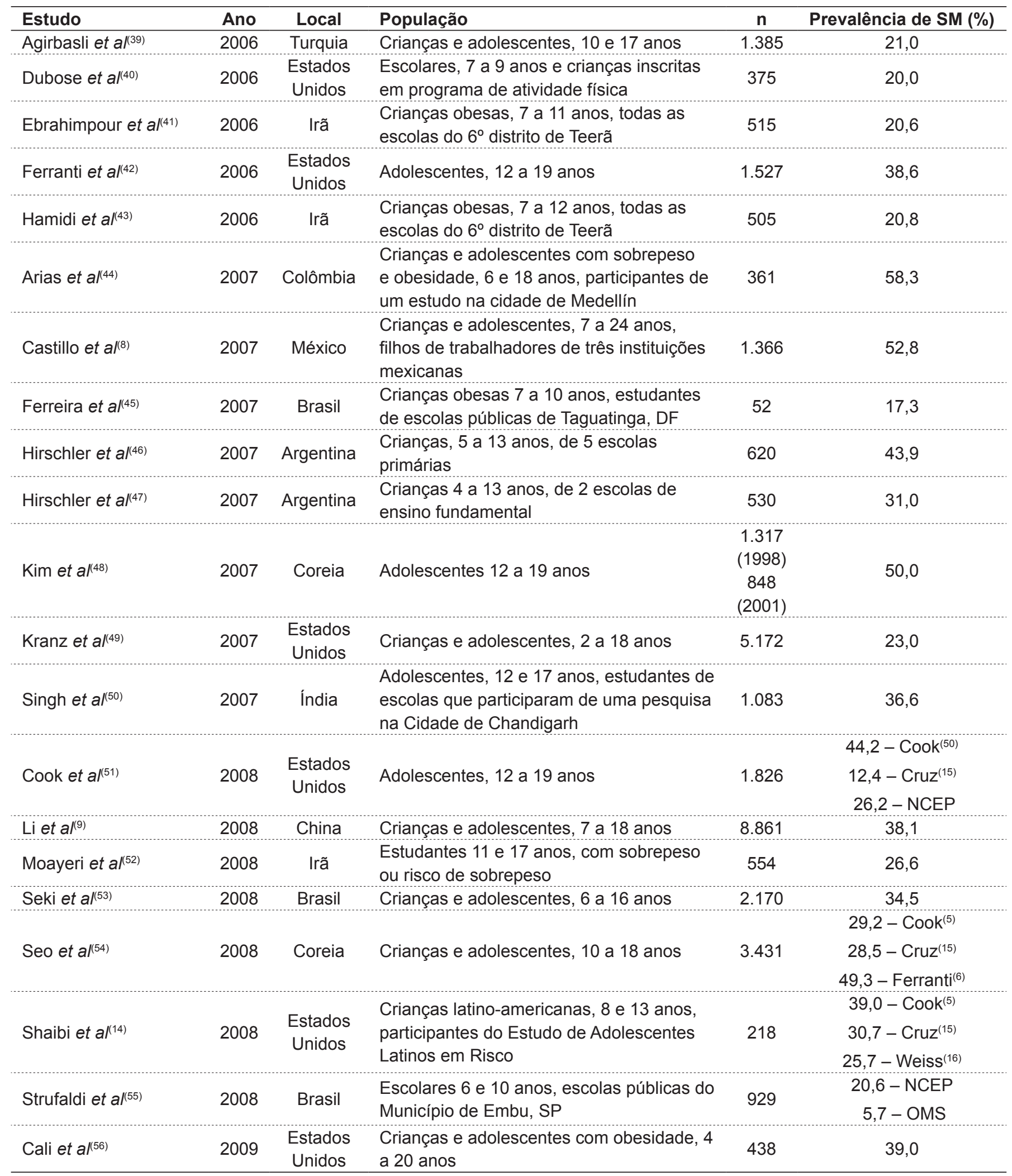

SM: síndrome metabólica; NCEP: National Cholesterol Education Program; OMS: Organização Mundial da Saúde; mod.: moderada. 
Tabela 3 - Componentes utilizados no diagnóstico de síndrome metabólica, crianças e adolescentes com sobrepeso ou obesidade, revisão da literatura, 2003-2009

\begin{tabular}{lccc}
\hline \multirow{2}{*}{ Componentes } & \multicolumn{2}{c}{ Artigos } & $\begin{array}{c}\text { Prevalência } \\
\text { mediana de SM (\%) }\end{array}$ \\
\cline { 2 - 3 } TG, HDL, GJ, CC e PA & $\mathbf{n}$ & $\mathbf{( \% )}$ & 31,2 \\
IMC, TG, HDL, PA e TOG & 28 & $(61,0)$ & 29,0 \\
TG, HDL, TOG, CC e PA & 7 & $(15,2)$ & 27,3 \\
IMC, TG, HDL, PA e GJ & 5 & $(10,9)$ & 26,1 \\
IMC, homeostase anormal da glicose (insulina de jejum, glicemia de & 5 & $(10,9)$ & 34,5 \\
jejum ou TOG), PA e dislipidemia (TG, HDL ou CT) & 2 & $(4,3)$ & \\
TG, HDL, CC, PA e homeostase anormal da glicose (GJ, TOG ou & 1 & $(2,2)$ & 18,6 \\
presença de diabetes melito) & 1 & $(2,2)$ & 34,0 \\
TG, HDL, HOMA-IR, CC e PA & 1 & $(2,2)$ & 30,0 \\
IMC, dislipidemia (TG, HDL ou CT), hiperinsulinemia e/ou IG & 1 & $(2,2)$ & 10,4 \\
TG, HDL,PA, relação entre gordura abdominal e nos membros & 1 & 20,6 \\
inferiores & 1 & $(2,2)$ & 5,7 \\
IMC, TG, HDL, IG e PA & 1 & $(2,2)$ & \\
IMC, TG, HDL, HOMA-IR e PA & & \\
\hline
\end{tabular}

TG: triglicerídeos; HDL: lipoproteína de alta densidade; GJ: glicemia de jejum; CC: circunferência da cintura; PA: pressão arterial; IMC: índice de massa corporal; TOG: tolerância oral à glicose; CT: colesterol total; IG: intolerância à glicose.

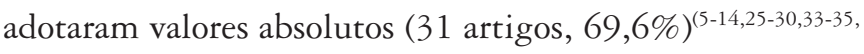
$38,40,42,44,45,48-50,52-55)$. Vale ressaltar que alguns autores estratificaram o HDL-c segundo sexo: Quintos et al(7) utilizaram 40 e $50 \mathrm{mg} / \mathrm{dL}$ como pontos de corte no sexos masculino e feminino, respectivamente, enquanto Castillo et al ${ }^{(8)}$ e Li et $a l^{(9)}$ aplicaram, respectivamente, 45 e $50 \mathrm{mg} / \mathrm{dL}$.

Uma importante divergência foi também observada em relação aos triglicerídeos séricos, tendo 29 autores utilizado limites que variaram de 100 a $150 \mathrm{mg} / \mathrm{dL}^{(5-14,25-28,30,34,35,38,40-}$ 42,48-50,52-55). Somente Atabek et al ${ }^{(10)}$ estabeleceram pontos segundo a faixa etária: $105 \mathrm{mg} / \mathrm{dL}$ até dez anos de idade e $136 \mathrm{mg} / \mathrm{dL}$ para maiores de dez anos.

A classificação da pressão arterial em percentil, por idade e estatura da criança, foi verificada em $39 \operatorname{artigos}(85 \%)^{(5,8,10,12-}$ 16,18,24,26-28,30-33,35-39,41-56), mas dois trabalhos estabeleceram os limites de pressão arterial em valores absolutos: 130x85 $\mathrm{mmHg}{ }^{(7)} ; 120 x 70 \mathrm{mmHg}$ e $130 x 80 \mathrm{mmHg}^{(11)}$.

Já o emprego da circunferência de cintura não apresentou grande divergência, com 28 artigos ${ }^{(5,6,8,9,11-15,25,27-30,35,38,40-43}$, 46-50,53,54) estabelecendo o uso dos percentis 75 e 90 como limites dessa variável. No entanto, novamente, Quintos et al ${ }^{(28)}$ empregaram valores absolutos: $102 \mathrm{~cm}$ para meninos e $88 \mathrm{~cm}$ para meninas. Somente um estudo analisou essa variável por meio de valores limítrofes de desvio padrão $(>1,3)$ como indicativo de excesso de gordura corporal, tendo como referência uma curva holandesa ${ }^{(7)}$.

Resumindo, os pontos de corte mais utilizados para as variáveis empregadas na definição de SM na infância e adolescência foram: HDL-c $<40 \mathrm{mg} / \mathrm{L}(20 \text { artigos })^{(5,7,11-}$
14,25-29,35,38,44,48-50,52,54,55), triglicerídeos séricos >110mg/L (19 artigos) $)^{(5,12,13,14,25,26,27,28,34,35,38,40,44,45,48,49,50,52,54)}$, glicemia $>110 \mathrm{mg} / \mathrm{L}$ (21 artigos) $)^{(5-10,13,14,25,30,34-38,40,42,48,50,52,54)}$, pressão

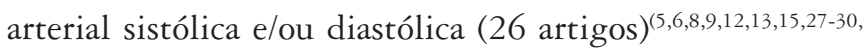
$34,35,37,38,40-44,47-50,53,54)$ e CC acima do percentil 90 (19 artigos) (5,11,13-15,25,27-29,35,38,40,41,43,48-50,53,54) e IMC superior aos percentis 95 ou 97 (13 artigos) $)^{(10,16,24,26,31,32,36,37,39,44,45,52,56)}$.

Analisando as distribuições de cada componente separadamente, as alterações mais prevalentes foram verificadas na CC, HDL-c e triglicerídeos, enquanto as menos prevalentes foram observadas em relação ao colesterol e a glicose (Tabela 4).

\section{Discussão}

O número expressivo de estudos existentes sobre SM na infância e adolescência é sugestivo de sua crescente relevância, sendo cada vez mais discutidos a definição e os parâmetros clínicos mais adequados para identificar crianças e adolescentes sob risco. A distribuição dos artigos realizados em diferentes populações no mundo pode denotar a preocupação mundial com a obesidade e suas consequentes alterações metabólicas.

A grande variabilidade nas prevalências encontradas poderia ser explicada pela divergência nos componentes da definição empregada, como nos pontos de corte adotados. Esse aspecto pode ser verificado por autores que utilizaram mais de um critério na análise de uma mesma amostra populacional. Bueno et $a^{(12)}$ e Burrows et al ${ }^{(13)}$, que se basearam nas propostas estabelecidas por Cook et al ${ }^{(5)}$ e Ferranti et al $l^{(6)}$, observaram uma variação entre as prevalências na ordem de 
$40 \%$. Já Shaibi et al ${ }^{(14)}$ utilizaram três propostas diferentes (Cook et $a l^{(5)}$, Cruz et $a l^{(15)}$ e Weiss et $a l^{(16)}$ ), tendo as prevalências variado entre 25,7 a 39,0\%.

A presença de três ou mais componentes para o diagnóstico da SM estabelecida em praticamente todos os artigos poderia decorrer do fato de que os critérios adotados para crianças se baseiam nas propostas de definição de SM para adultos.

Hipertrigliceridemia, redução do HDL-c, hiperglicemia de jejum, obesidade abdominal (CC) e hipertensão arterial são os componentes utilizados pelo NCEP/ATPIII na definição da SM em adultos para uma aplicação clínica direta, por se tratarem de critérios mais específicos. Provavelmente, por tal razão, essa foi a proposta mais utilizada pelos pesquisadores no diagnóstico da SM em crianças e adolescentes.

A CC tem sido recomendada por diversas organizações (OMS, NCEP, IDF) como indicativo de adiposidade visceral em adultos. Entretanto, existe uma grande limitação de seu uso em crianças e adolescentes, pois ela é dependente de comparação com uma curva de distribuição representativa de uma população. Sendo assim, se faz necessária a construção de curvas representativas de uma determinada população, levando-se em consideração estatura, idade e etnia. Vale ressaltar que o Brasil possui uma curva, mas esta é restrita à idade de dez anos e representativa unicamente da população infantil de Florianópolis (SC). Outra limitação da CC diz respeito à técnica para obter a medida, que muitas vezes não é descrita, ou se adota a técnica de circunferência abdominal (cicatriz umbilical). Além disso, é uma medida que pode apresentar pequena reprodutibilidade e grande variabilidade inter e intraobservador, na ausência de protocolo para coleta e treinamento prévio ${ }^{(17)}$.

Esses problemas no uso da CC levaram alguns pesquisadores a utilizarem o IMC, que se baseia em medidas simples, de fácil obtenção (peso e estatura) e com referência e pontos de corte bem estabelecidos. As recentes curvas de crescimento de zero a cinco anos da OMS, de 2006, foram elaboradas com população representativa de diversas partes do mundo. Já as curvas da OMS de 2007, de cinco a 19 anos, foram uma reconstrução das curvas do National Center for

Quadro 1 - Pontos de corte dos componentes da síndrome metabólica, crianças e adolescentes com sobrepeso ou obesidade, revisão da literatura, 2003-2009

\begin{tabular}{|lcc|}
\hline Componente & Ponto de corte & N $^{\circ}$ estudos \\
\hline CT & $>$ P95 & 2 \\
\hline & $<35 \mathrm{mg} / \mathrm{dL}$ & 2 \\
HDL & $<38 \mathrm{mg} / \mathrm{dL}$ & 1 \\
& $<40 \mathrm{mg} / \mathrm{dL}$ & 20 \\
& $<45 \mathrm{mg} / \mathrm{dL}$ & 1 \\
& $<50 \mathrm{mg} / \mathrm{dL}$ & 5 \\
& $<\mathrm{P} 5$ & 13 \\
& $<\mathrm{P} 10$ & 3 \\
& $>100 \mathrm{mg} / \mathrm{dL}$ & 6 \\
TG & $>110 \mathrm{mg} / \mathrm{dL}$ & 19 \\
& $>120 \mathrm{mg} / \mathrm{dL}$ & 1 \\
& $>130 \mathrm{mg} / \mathrm{dL}$ & 2 \\
& $>150 \mathrm{mg} / \mathrm{dL}$ & 1 \\
& $>\mathrm{P} 90$ & 6 \\
\multirow{2}{*}{ GJ } & $>\mathrm{P} 95$ & 10 \\
\hline TOG & $>100 \mathrm{mg} / \mathrm{dL}$ & 18 \\
\hline \multirow{2}{*}{ PA } & $>110 \mathrm{mg} / \mathrm{dL}$ & 21 \\
\hline \multirow{2}{*}{ CC } & $140-200 \mathrm{mg} / \mathrm{dL}$ & 9 \\
\hline \multirow{3}{*}{ IMC } & $>\mathrm{P} 90$ & 22 \\
& $>\mathrm{P} 95$ & 17 \\
\hline
\end{tabular}

CT: colesterol total; HDL: lipoproteína de alta densidade; TG: triglicerídeos; GJ: glicemia de jejum; TOG: tolerância oral à glicose; PA: pressão arterial; CC: circunferência da cintura; IMC: índice de massa corporal.

Tabela 4 - Prevalência mediana de alterações metabólicas descritas como associadas à síndrome metabólica no sobrepeso e obesidade em crianças e adolescentes, revisão da literatura, 2003-2009

\begin{tabular}{lccc}
\hline Componente & Prevalência mínima (\%) & Prevalência máxima (\%) & Prevalência mediana (\%) \\
\hline CC & 10,7 & 98,1 & 68,5 \\
HDL-C & 5,2 & 85,4 & 33,2 \\
TG & 7,5 & 81,2 & 34,2 \\
PA & 7,1 & 58,7 & 24,7 \\
CT & 8,9 & 36,7 & 15,4 \\
TOG & 3,6 & 27,0 & 10,3 \\
GJ & 0 & 28,3 & 5,8 \\
\hline
\end{tabular}

CC: circunferência da cintura; HDL-c: lipoproteína de alta densidade; TG: triglicerídeos; PA: pressão arterial; CT: colesterol total; TOG: tolerância oral à glicose; GJ: glicemia de jejum. 
Health Statistics (NCHS), em 1977, que utiliza os dados das primeiras curvas, suplementados com dados das curvas da OMS para menores de cinco anos, por meio de metodologia estatística semelhante à utilizada na construção da referência da OMS. Essas curvas têm, portanto, se mostrado muito úteis na comparação dos estudos. Entretanto, a não utilização do IMC como componente da SM poderia se justificar pelo fato desse índice nem sempre apresentar correlação direta com a adiposidade na população adulta. Vale ressaltar que, na população infantil, dificilmente o IMC elevado não está associado ao aumento de gordura corporal.

Já Mimoun et al ${ }^{(18)}$ utilizaram uma razão entre gordura abdominal e membros inferiores maior que 0,85 como indicativo de adiposidade, não ocorrendo, entretanto, detalhamento da técnica na coleta das medidas que geraram essa razão e não sendo justificada sua utilização. Devido ao ponto de corte estabelecido, este componente equivaleria à relação cintura-quadril (RCQ), descrita pela OMS em 1999, mas não mais considerada como critério diagnóstico da SM. A RCQ nem sempre reflete acúmulo de gordura abdominal, podendo também indicar uma pequena área muscular no quadril. Adicionalmente, pode não sinalizar risco, apresentando valores reduzidos em indivíduos com acúmulo de gordura abdominal, acompanhado de grande acúmulo de gordura no quadril ${ }^{(19)}$. Nesse contexto, persiste o questionamento sobre qual o critério antropométrico mais prático e fácil de ser utilizado em crianças, que expresse tanto o excesso de gordura como sua localização.

Todos os artigos incluíram alterações de perfil lipídico (hipertrigliceridemia e diminuição do HDL-c), da homeostase da glicose (hiperglicemia de jejum ou intolerância à glicose) e hipertensão arterial como componentes da SM. Esse fato poderia ser justificado pela alteração do metabolismo lipídico, bem como o da glicose e o aumento da pressão arterial advirem do excesso de gordura corporal. $\mathrm{O}$ tecido adiposo é um órgão imuno-endócrino-metabólico, secretando adipocinas e citocinas pró-inflamatórias (TNF- $\alpha$, IL-6 e IL-1) capazes de induzir a resistência à insulina, com hiperinsulinemia compensatória. Além disso, os adipócitos viscerais são insulino-resistentes, o que leva ao aumento da lipólise mediada por catecolaminas. A não inibição da lipólise, devido à hiperinsulinemia, acarreta maior liberação de ácidos graxos livres. Esses são transportados para a veia porta e, ao chegarem ao fígado, podem causar alterações no metabolismo lipídico ${ }^{(20)}$.

A partir dos ácidos graxos livres, o fígado sintetiza triglicerídeos que são agrupados formando a lipoproteína de muito baixa densidade (VLDL-c). Pela ação da proteína transferidora de ésteres de colesterol, a VLDL-c permuta triglicerídeos por ésteres de colesterol da LDL-c e HDL-c. As lipases atuam na LDL-c rica em triglicerídeos formando LDL-c pequenas e densas (mais aterogênicas). Já a HDL-c perde moléculas de apolipoproteína A-1, diminuindo sua capacidade de fazer transporte reverso de colesterol. Essa série de eventos produz lipoproteínas ricas em triglicerídeos, mais aterogênicas, e diminuição da concentração de HDL-c ${ }^{(20)}$.

A resistência à insulina também é responsável pelo aumento da pressão arterial por meio da elevação da frequência cardíaca e ativação adrenérgica, além de diminuir a produção de óxido nítrico, que é um potente vasodilatador ${ }^{(21)}$. Diante da resistência à insulina como alteração metabólica precursora, muito se discute sobre qual seria o melhor critério para seu diagnóstico. Esse fato pode ser verificado pela divergência dos estudos entre o uso da glicemia de jejum, a tolerância oral à glicose ou o índice HOMA-IR.

O uso da tolerância oral à glicose ou o índice HOMA-IR baseia-se no fato de que os valores de glicemia de jejum dificilmente se apresentam alterados nessa população, devido à capacidade de homeostase metabólica dessa molécula. Quando a insulina está aumentada, a produção de glicose por meio da gliconeogênese é inibida ${ }^{(20)}$, o que pode ser inferido pela baixa prevalência da hiperglicemia de jejum. O índice HOMA-IR, criado por Turner et al, é um modelo matemático que prediz a sensibilidade à insulina pela medida da glicemia e insulina de jejum. Tal método foi chamado de HOMA e dele se extraem dois índices (HomaIR e Homabeta), que visam traduzir a sensibilidade à insulina e a capacidade secretória de célula beta, ou, em outras palavras, a resistência à insulina e a função de célula beta ${ }^{(21)}$. No entanto, existe uma grande dificuldade na prática clínica em se utilizar a tolerância oral à glicose ou o HOMA-IR, pois se tratam de exames caros e de coleta mais difícil em crianças. Além disso, não existe consenso a partir de que valores dessas variáveis há aumento de risco à saúde em crianças e adolescentes.

Dentro do perfil lipídico, o colesterol sérico só foi incluído em dois artigos. Isso se deve ao fato dessa variável não ser considerada componente da SM em adultos, sendo a elevação dos triglicerídeos e a redução do HDL-c consideradas as principais alterações no perfil lipídico advindas da resistência à insulina ${ }^{(20)}$. Entretanto, a utilização do colesterol pode ser justificada por se tratar de um fator de risco bem estabelecido para doenças cardiovasculares, relacionado à obesidade. Esse fato foi observado no Bogalusa Heart Study, no qual crianças obesas apresentaram um risco 2,4 vezes 
maior de hipercolesterolemia em relação a crianças de peso normal $^{(4)}$.

Apesar da unanimidade na utilização da HDL-c e triglicerídeos por sua incontestável relação com risco de doenças cardiovasculares, houve muita divergência nos pontos de corte utilizados, devido à dificuldade em se estabelecer um limite de normalidade, a partir do qual há aumento do risco de morbidade. Dessa forma, os autores estabeleceram limites ora em percentis de uma distribuição, ora adotando valores absolutos. Apesar de ter havido diferenciação dos valores de HDL-c por sexo, este é bastante questionável em crianças, pois não são encontradas diferenças estatisticamente significantes entre meninos e meninas. Já nos adultos, essa diferenciação se justificaria pela relação entre a HDL-c e os níveis de 17-beta-estradiol no sexo feminino. A realização da estratificação talvez não se justifique na população infantil, pois as meninas não têm atividade hormonal expressiva e alguns autores sugerem que a opção mais adequada seria a estratificação dos níveis de HDL-c segundo estágio de maturação sexual ${ }^{(22)}$.

A importância da inclusão da pressão arterial como componente da SM, encontra-se bem estabelecida, já sendo validados os limites de corte nas distribuições para crianças, nas quais os mesmos são estratificados por sexo, idade e percentil de estatura/idade. Dessa forma, não parece adequado estabelecer o diagnóstico da hipertensão arterial por meio de limites absolutos, semelhantes aos usados na população

\section{Referências bibliográficas}

1. Chiarelli F, Marcovecchio ML. Insulin resistance and obesity in childhood. EurJ Endocrinol 2008;159 (Suppl 1):S67-74.

2. De Ferranti SD, Osganian SK. Epidemiology of paediatric metabolic syndrome and type 2 diabetes mellitus. Diab Vasc Dis Res 2007;4:285-96.

3. Chen W, Berenson GS. Metabolic syndrome: definition and prevalence in children. J Pediatr (Rio J) 2007;83:3-11.

4. Freedman DS, Dietz WH, Srinivasan SR, Berenson GS. The relation of overweight to cardiovascular risk factors among children and adolescents: The Bogalusa Heart Study. Pediatrics 1999;103:1175-82.

5. Cook S, Weitzman M, Auinger P, Nguyen M, Dietz WH. Prevalence of a metabolic syndrome phenotype in adolescents: findings from the third national health and nutrition examination survey, 1988-1994. Arch Pediatr Adolesc Med 2003;157:821-7.

6. Ferranti SD, Gauvreau K, Ludwig DS, Neufeld EJ, Newburger JW, Rifai N. Prevalence of the metabolic syndrome in American adolescents: findings from the third national health and nutrition examination survey. Circulation 2004;110:2494-7.

7. Quintos JB, Muzumdar H, George M, Mercado AB, Lu H, Sternberg A et al. The prevalence of metabolic syndrome in inner city obese African-American youth. Pediatr Endocrinol Rev 2006;3 (Suppl 4):571-5.

8. Halley Castillo E, Borges G, Talavera JO, Orozco R, Vargas-Alemán C, HuitrónBravo $\mathrm{G}$ et al. Body mass index and the prevalence of metabolic syndrome adulta, como realizado em duas investigações incluídas nesta revisão ${ }^{(7,11)}$.

Apesar da grande variabilidade, a prevalência relatada de $\mathrm{SM}$ em crianças e adolescentes com sobrepeso e obesidade foi elevada, em torno de 30\%. Embora existam questionamentos da sua existência nessa população ${ }^{(23)}$, os resultados ressaltam a importância das alterações metabólicas em crianças e adolescentes, indicando aumento dos riscos de morbidade por doenças crônicas na vida adulta.

\section{Conclusão}

A prevalência da SM variou de 2,1 a $58,3 \%$ e o critério diagnóstico mais utilizado, porém sem consenso nos pontos de corte, foi semelhante ao do NCEP: hipertrigliceridemia, redução do HDL-c, hiperglicemia de jejum, obesidade abdominal e hipertensão arterial. Os pontos de corte mais adotados foram: HDL-c $\geq 40 \mathrm{mg} / \mathrm{L}$, triglicerídeos $\geq 110 \mathrm{mg} / \mathrm{L}$, glicemia $\geq 110 \mathrm{mg} / \mathrm{L}$, pressão arterial sistólica e/ou diastólica e $\mathrm{CC} \geq \mathrm{p} 90$, e IMC $\geq \mathrm{p} 95 / 97$. As alterações mais frequentemente relatadas nos artigos revisados ocorreram em relação à CC, HDL-c e triglicerídeos, enquanto o colesterol e a glicose foram as menos prevalentes. Apesar da obesidade e de suas consequências metabólicas serem cada vez mais prevalentes em crianças e adolescentes, não existe consenso nos critérios utilizados para diagnosticar a SM, seja quanto à definição de seus componentes ou aos pontos de corte nas respectivas distribuições. among children and adolescents in two Mexican populations. J Adolesc Health 2007;40:521-6.

9. Li Y, Yang X, Zhai F, Piao J, Zhao W, Zhang J et al. Childhood obesity and its health consequence in China. Obes Rev 2008; 9 (Suppl 1):S82-6.

10. Atabek ME, Pirgon O, Kurtoglu S. Prevalence of metabolic syndrome in obese Turkish children and adolescents. Diabetes Res Clin Pract 2006;72:315-21.

11. Yoshinaga M, Tanaka S, Shimago A, Sameshima K, Nishi J, Nomura Y et al. Metabolic syndrome in overweight and obese Japanese children. Obes Res 2005;13:1135-40.

12. Bueno G, Bueno O, Moreno LA, García R, Tresaco B, Garagorri JM et al. Diversity of metabolic syndrome risk factors in obese children and adolescents. J Physiol Biochem 2006;62:125-34.

13. Burrows AR, Leiva BL, Weistaub G, Ceballos SX, Gattas ZV, Lera ML et al. Síndrome metabólico en niños y adolescentes: asociación con sensibilidad insulínica y con magnitud y distribución de la obesidad. Rev Med Chile 2007;135:174-81.

14. Shaibi GQ, Goran MI. Examining metabolic syndrome definitions in overweight Hispanic youth: a focus on insulin resistance. J Pediatr 2008;152:171-6.

15. Cruz ML, Weigensberg MJ, Huang TT, Ball G, Shaibi GQ, Goran MI. The metabolic syndrome in overweight Hispanic youth and the role of insulin sensitivity. J Clin Endocrinol Metab 2004;89:108-13.

16. Weiss R, Dziura J, Burgert TS, Tamborlane WV, Taksali SE, Yeckel CW et al. 
Obesity and the metabolic syndrome in children and adolescents. N Engl $\mathrm{J}$ Med 2004;350:2362-74.

17. Mason C, Katzmarzyk PT. Variability in waist circumference measurements according to anatomic measurement site. Obesity (Silver Spring) 2009;17:178995.

18. Mimoun E, Aggoun Y, Pousset M, Dubern B, Bouglé D, Girardet JP et al. Association of arterial stiffness and endothelial dysfunction with metabolic syndrome in obese children. J Pediatr 2008;153:65-70.

19. Visscher TL, Seidell JC, Molarius A, van der Kuip D, Hofman A, Witteman JC. A comparison of body mass index, waist-hip ratio and waist ircumference as predictors of all-cause mortality among the elderly: the Rotterdam study. Int $\mathrm{J}$ Obes Relat Metab Disord 2001;25:1730-5.

20. Fulop T, Tessier D, Carpentier A. The metabolic syndrome. Pathol Biol (Paris) 2006;54:375-86.

21. Geloneze B, Tambascia MA. Laboratorial evaluation and diagnosis of insulin resistance. Arq Bras Endocrinol Metab 2006;50:208-15.

22. Lima SC, Arrais RF, Almeida MG, Souza ZM, Pedrosa LF. Plasma lipid profile and lipid peroxidation in overweight or obese children and adolescents. $J$ Pediatr (Rio J) 2004;80:23-8

23. Oliveira CL, Mello MT, Cintra IP, Fisberg M. Obesity and metabolic syndrome in infancy and adolescence. Rev Nutr Campinas, 2004;17:237-45.

24. Boney CM, Verma A, Tucker R, Vohr BR. Metabolic syndrome in childhood: association with birth weight, maternal obesity, and gestational diabetes mellitus. Pediatrics 2005;115:e290-6.

25. Esmaillzadeh A, Mirmiran P, Azadbakht L, Etemadi A, Azizi F. High prevalence of the metabolic syndrome in Iranian adolescents. Obesity (Silver Spring) 2006;14:377-82.

26. López-Capapé M, Alonso M, Colino E, Mustieles C, Corbatón J, Barrio R. Frequency of the metabolic syndrome in obese Spanish pediatric population. Eur J Endocrinol 2006;155:313-9.

27. Buff CG, Ramos E, Souza FI, Sami RO. Freqüência de síndrome metabólica em crianças e adolescentes com sobrepeso e obesidade. Rev Paul Pediatr 2007;25:221-6.

28. Ceballos T, Siguero JPL, Ortiz AJ. Prevalencia del síndrome metabólico y sus componentes en niños y adolescentes con obesidad. An Pediatr (Barc) 2007;67:352-61.

29. Dhuper S, Cohen HW, Daniel J, Gumidyala P, Agarwalla V, St Victor R et al. Utility of the modified ATP III defined metabolic syndrome and severe obesity as predictors of insulin resistance in overweight children and adolescents: a cross-sectional study. Cardiovasc Diabetol 2007;6:4.

30. Serap S, Mevlüt B, Inanç C, Ender S. Metabolic syndrome in childhood obesity. Indian Pediatr 2007;44:657-62.

31. Calcaterra V, Klersy C, Muratori T, Telli S, Caramagna C, Scaglia F et al. Prevalence of metabolic syndrome (MS) in children and adolescents with varying degrees of obesity. Clin Endocrinol (Oxf) 2008;68:868-72.

32. Caceres M, Teran CG, Rodriguez S, Medina M. Prevalence of insulin resistance and its association with metabolic syndrome criteria among Bolivian children and adolescents with obesity. BMC Pediatr 2008;8:31.

33. Cizmecioğlu FM, Hatun S, Kalaça S. Metabolic syndrome in obese Turkish children and adolescents: comparison of two diagnostic models. Turk J Pediatr 2008;50:359-65.

34. Kolsgaard ML, Andersen LF, Tonstad S, Brunborg C, Wangensteen T, Joner G. Ethnic differences in metabolic syndrome among overweight and obese children and adolescents: the Oslo Adiposity Intervention Study. Acta Paediatr 2008;97:1557-63.

35. Lee S, Bacha F, Gungor N, Arslanian S. comparison of different definitions of pediatric metabolic syndrome: relation to abdominal adiposity, insulin resistance, adiponectin, and inflammatory biomarkers. J Pediatr 2008; 152:177-84.

36. Sen Y, Kandemir N, Alikasifoglu A, Gonc N, Ozon A. Prevalence and risk factors of metabolic syndrome in obese children and adolescents: the role of the severity of obesity. Eur J Pediatr 2008;167:1183-9.

37. Rodríguez-Morán $M$, Salazar-Vásquez B, Violante R, Guerrero-Romero $F$. Metabolic syndrome among children and adolescents aged 10-18 years. Diabetes Care 2004;27:2516-7.

38. Braunschweig CL, Gomez S, Liang H, Tomey K, Doerfler B, Wang Y et al. Obesity and risk factors for the metabolic syndrome among low-income, urban, African American schoolchildren: the rule rather than the exception? Am J Clin Nutr 2005;81:970-5.

39. Agirbasli M, Cakir S, Ozme S, Ciliv G. Metabolic syndrome in Turkish children and adolescents. Metabolism 2006;55:1002-6.

40. DuBose KD, Stewart EE, Charbonneau SR, Mayo MS, Donnelly JE. Prevalence of the metabolic syndrome in elementary school children. Acta Paediatr 2006;95:1005-11.

41. Ebrahimpour P, Fakhrzadeh $H$, Pourebrahim R, Hamidi A, Larijani B. Metabolic Syndrome and Related Insulin Levels in Obese Children. Metab Syndr Relat Disord 2006;4:172-8.

42. de Ferranti SD, Gauvreau K, Ludwig DS, Newburger JW, Rifai N. Inflammation and changes in metabolic syndrome abnormalities in US adolescents: findings from the 1988-1994 and 1999-2000 National Health and Nutrition Examination Surveys. Clin Chem 2006;52:1325-30.

43. Hamidi A, Fakhrzadeh H, Moayyeri A, Heshmat R, Ebrahimpour P, Larijani B. Metabolic syndrome and leptin concentrations in obese children. Indian J Pediatr 2006;73:593-6.

44. Arias AR, Agudelo Ochoa GM. Prevalencia de algunos componentes del síndrome metabólico en escolares y adolescentes con sobrepeso y obesidad. Perspect Nutr Hum 2007;9:11-22.

45. Ferreira AP, Oliveira CE, França NM. Metabolic syndrome and risk factors for cardiovascular disease in obese children: the relationship with insulin resistance (HOMA-IR). J Pediatr (Rio J) 2007;83:21-6.

46. Hirschler V, Roque MI, Calcagno ML, Gonzalez C, Aranda C. Maternal waist circumference and the prediction of children's metabolic syndrome. Arch Pediatr Adolesc Med 2007;161:1205-10.

47. Hirschler V, Maccallini G, Calcagno M, Aranda C, Jadzinsky M. Waist circumference identifies primary school children with metabolic syndrome abnormalities. Diabetes Technol Ther 2007;9:149-57.

48. Kim HM, Park J, Kim HS, Kim DH. Prevalence of the metabolic syndrome in Korean adolescents aged 12-19 years from the Korean National Health and Nutrition Examination Survey 1998 and 2001. Diabetes Res Clin Pract 2007;75:111-4.

49. Kranz S, Mahood LJ, Wagstaff DA. Diagnostic criteria patterns of U.S. children with Metabolic Syndrome: NHANES 1999-2002. Nutr J 2007;6:38.

50. Singh R, Bhansali A, Sialy R, Aggarwal A. Prevalence of metabolic syndrome in adolescents from a north Indian population. Diab Med 2007;24:195-9.

51. Cook S, Auinger P, Li C, Ford ES. Metabolic syndrome rates in United States adolescents, from the National Health and Nutrition Examination Survey, 1999-2002. J Pediatr 2008;152:165-70.

52. Moayeri H, Rabbani A, Keihanidoust ZT, Bidad K, Anari S. Overweight adolescents: a group at risk for metabolic syndrome (Tehran adolescent obesity study). Arch Iran Med 2008;11:10-5.

53. Seki M, Matsuo T, Carrilho AJ. Prevalence of metabolic syndrome and associated risk factors in Brazilian schoolchildren. Public Health Nutr 2009;12:947-52.

54. Seo SJ, Lee HY, Lee SW. The prevalence of the metabolic syndrome in Korean children and adolescents: comparisons of the criteria of Cook et al. Yonsei Med J 2008;49:563-72.

55. Strufaldi MW, Silva EM, Puccini RF. Metabolic syndrome among prepubertal Brazilian schoolchildren. Diab Vasc Dis Res 2008;5:291-7.

56. Cali AM, Caprio S. Ectopic fat deposition and the metabolic syndrome in obese children and adolescents. Horm Res 2009;71 (Suppl 1):S2-7. 